\title{
Von Warschau nach New York. Die poetischen Passagen der Dichterin Kadya Molodowsky
}

W roku 1935 udaje się Mołodowskiej wyemigrować z Warszawy do Nowego Jorku. Poezja jej od samego początku wyraża uczucie obcości i wygnania. Debiut literacki w roku 1920 jest początkiem twórczości ściśle związanej z religijną tradycją i świeckim ,yidishkayt‘. Mołodowska tematyzuje doświadczenia ubóstwa i antysemityzmu oraz przeżycia żydowskich kobiet rozdartych pomiędzy emancypacją a ścisłym związkiem z religijną tradycją. Powstała w USA poezja Mołodowskiej akcentuje głównie niezwykle trudną sytuację imigrantów: presję wielonarodowej społeczności, język jidysz jako symbol tradycji i tożsamości oraz grożącą utratę zbiorowej pamięci, która prowadzi do stopniowego niwelowania różnic zarówno kulturowych, jak i językowych.

1935 gelingt Molodowsky die Emigration von Warschau nach New York. Ihre Poesie verarbeitet schon von Beginn an Erfahrungen der Fremdheit und des Exils: 1920 bildet ihr Debüt den Auftakt für ein literarisches Werk, das eine enge Verbindung von religiöser Tradition und säkularer ,yidishkayt ‘ eingeht. Ihr Frühwerk thematisiert die Erfahrung von Armut und Antisemitismus, und auch diejenige von Frauen zwischen Emanzipation und Verbundenheit mit ihrer traditionellen Herkunft. Die in den USA entstandene Dichtung akzentuiert insbesondere die Schwellensituation der Immigranten: den Druck der Schmelztiegelgesellschaft, die jiddische Sprache als Trägerin von Tradition und Identität sowie den drohenden Verlust kollektiver Erinnerung durch eine Gesellschaftssituation, in der kulturelle wie sprachliche Differenzen allmählich eingeebnet werden.

Molodowsky emigrated from Warsaw to New York in 1935. Her poetry evokes the experiences of estrangement and exile. Her poetic debut in 1920 already foreshadows a literary life-work which seeks to establish a close connection between religious tradition and secular, yidishkayt'. Her earliest work also reflects social poverty and anti-Semitic terror, as well as the particular experiences affecting Jewish women, especially concerning the tensions they faced between the lure of emancipation and 
their religious family background. Molodowsky's American work tends to highlight the threshold situation confronting recent immigrants, the impact of "the melting pot" on their lives and consciousness. At the same time it explores the impact of assimilation on the Yiddish language as a medium of tradition and identity, as well as the inevitable loss of collective memory by a society which tends to level both cultural and linguistic differences.

Im Jahr 1935 gelang Kadya Molodowsky (1894-1975) die Emigration von Warschau nach New York. Im Mittelpunkt dieses Beitrages stehen ihre Lyrik und Prosa, entstanden aus der Erfahrung der osteuropäischen jüdischen Emigrantin zwischen 1935 und 1942, d.h. ein Jahr bevor die ersten Nachrichten vom systematischen Mord an den Juden durch die deutschen Nationalsozialisten Molodowskys Appartement an der New Yorker East Side erreichten. ${ }^{1}$ Diese Texte, die noch nicht explizit auf das Vernichtungsereignis reagieren, eröffnen Fragen in Hinsicht auf vielfältige Differenz- und Schwellenerfahrungen: kulturelle und sprachliche Differenzen wie Barrieren zwischen Juden und Nichtjuden in Osteuropa und Amerika, sexuelle Differenz innerhalb des jüdischen Lebens sowie Phänomene des Übergangs von einer Lebenswelt in die andere. Gerade die Emigrantinnen waren nicht nur mit dem Sprung in die unbekannte Kultur konfrontiert. In einer Zeit, die vom akuten Prozess markanter Umwandlungen der traditionellen Lebensweise markiert war, die BENJAMIN HARSHAV (1995:25) als „Jüdische Revolution“ bezeichnet, waren sie auch auf der Suche nach Orten in dem in Bewegung geratenen System der Geschlechterverhältnisse innerhalb der eigenen Kultur. Die verbreitete Formel von der doppelten Alterität der Immigrantin - als Frau und abseits der Mehrheitskultur - erscheint zu starr angesichts der wechselnden und neuen Allianzen, die alte Differenzierungen aufheben, vertikal zu den bestehenden verlaufen und in Binnenspannungen zueinander stehen. HELLERSTEIN (1995:67) spricht in diesem Zusammenhang von einem mehrfachen Exil und umschreibt damit zugleich die besondere Situation von Jiddisch schreibenden Autorinnen:

$1 \quad$ Molodowsky veröffentlichte in den 1960er und 70er Jahren sukzessive ihre autobiographischen Aufzeichnungen in dem Journal Svive [Umfeld], das sie selbst 1943 in New York gegründet hatte. Vgl. MoLodowsKy (1965-1974). Entlang dieser Notizen rekonstruiert KATHRYN HELLERSTEIN, dass die nationalsozialistischen Vernichtungspläne erst im Jahr 1943 ganz konkret in deren Bewusstsein gelangten, möglicherweise über Nachrichten vom Aufstand im Warschauer Ghetto und dessen Niederschlagung (HELLERSTEIN 1995:41). Den Vernichtungsereignissen widmet sich insbesondere Molodowskys Gedichtband Der meylekh dovid aleyn iz geblibn [König David allein ist geblieben, 1946]. 
She [a woman writing modern poetry in Yiddish - Ch.P.] had to define her poetry against both the Yiddish devotional culture of women and the Hebraic culture of traditional Judaism. She was also chastised by her male colleagues to distance herself from so-called female literary patterns and conform to male poetic standards, at which she was doubly bound to fail. By choosing to write poetry in Yiddish, she placed herself in a web of exclusion that exiled her self in the world at large and her gender and language within Judaism.

In diesen religiös-kulturell wie geschlechterspezifisch begründeten Spannungsfeldern finden sich jene Konstellationen von Sprache, Macht und Differenzen, welche die poetischen Passagen der Dichterin Molodowsky verarbeiten.

Molodowsky kam 1894 im weißrussischen Bereza Kartuska zur Welt. Dort erhielt sie zunächst eine traditionelle Erziehung durch ihre Großmutter, später wurde sie unterrichtet von ihrem Vater Isaac, einem Bewunderer Theodor Herzls und Anhänger der ,Haskalah', der Talmud und Hebräisch im ,Cheder ‘ unterrichtete. $^{2}$ Dieser eröffnete seiner Tochter im Kreise seiner ansonsten männlichen Schüler den Zugang zu den Texten der jüdischen Tradition und machte sie zugleich mit der traditionellen und zeitgenössischen jiddischen Literatur, aber auch darüber hinaus mit einem umfangreichen säkularen Wissen vertraut. Nicht zuletzt diese Ausnahmesituation, als einziges Mädchen in einer männlich dominierten Bildungssozialisation das Privileg einer intellektuellen Grundausbildung zu erhalten, weckte Molodowskys kritisches Bewusstsein gegenüber sozialer Ungleichheit und politischer Unterdrückung. Die nachhaltige Förderung ermöglichte ihr das Examen am Hebräischen Lehrer-Seminar in Warschau und ließ gleichzeitig ihre literarischen Bestrebungen erwachen, die jedoch erst ein paar Jahre später in Publikationen münden sollten. Mit Beginn des Ersten Weltkrieges begann für Molodowsky eine unruhige Zeit. Sie besuchte gerade ihre Eltern in Bereza, als die Stadt sich mit jüdischen Bewohnern der benachbarten Städte und Dörfer füllte, die vor der näherrückenden Front flüchteten. Die Menschen wurden aus ihren gewohnten Umgebungen gerissen, damit einher gingen Obdachlosigkeit und Armut. Die avancierten Bildungsprojekte für Kinder, wie sie zu der Zeit in Warschau entwickelt wurden und an denen auch Molodowksy enthusiastisch teilhatte, konnten nicht wie geplant umgesetzt werden. So hielt sie sich während der kommenden Jahre an wechselnden Orten auf, arbeitete in einem

2 Die biographischen Angaben zu Molodowsky folgen weitgehend HELLERSTEIN (1995:17-60), KLEPFISZ (1994), FishMAN GONSHOR (1997:15-27) sowie RAVITSH (1945:122-124). 
Warschauer Waisenhaus, unterrichtete im ukrainischen Romny sowie im russischen Saratow und betreute schließlich nach einem kurzen Aufenthalt in Odessa elternlose Kinder in Kiew. Die meisten von ihnen waren während der Kiew-Pogrome des Jahres $1919^{3}$ aus den umliegenden Orten vertrieben worden. Es war diese Zeit der existentiellen Bedrohung, in der sich Molodowskys literarische und politische Ambitionen entfalteten. In den ersten zwei Jahrzehnten, nachdem Kiew sowjetisch geworden war, entwickelte sich die Stadt zu einem der jüdischen intellektuellen Zentren, in denen eine intensive literarische Aufbruchstimmung herrschte. Molodowsky begann, die an allen ihren Aufenthaltsorten der vergangenen Jahre erfahrene Armut, den Hunger und den jüngst erlebten antisemitischen Terror in Poesie zu verarbeiten. Ihr Debüt im Jahr 1920, die Veröffentlichung zweier Gedichte in einer Lyrik-Anthologie ${ }^{4}$, lanciert u. a. von David Bergelson, bildete den Auftakt für ein literarisches Werk, angefüllt mit Romanen, Essays, Dramen, einer großen Menge an Kinderliteratur und vor allem Lyrik.

Schon früh hatte sie begonnen, sich von der Gedanken- und Erziehungswelt ihrer Herkunft zu entfernen, ohne dass das kollektive religiöse Gedächtnis je aus ihrer Bewusstseins- und Gedankenwelt verschwunden wäre. In ihren Gedichten jener ersten Jahre wandelt sich die erfahrene Dreifachmarginalisierung als weibliche Angehörige eines ärmlichen jüdischen, großteils orthodoxen Milieus von der individuellen Sozialerfahrung zum Teil des kollektiven Bewusstseins einer sozial, ökonomisch und politisch unterdrückten Minderheit. Zurück in Warschau publizierte sie eine Reihe von Texten, die die inneren Kämpfe und Sehnsüchte osteuropäischer jüdischer Frauen auf der Schwelle der Emanzipation von den tradierten Weiblichkeitsrollen, oftmals in der Figur der Mutter verkörpert, thematisieren, wie das folgende Gedicht aus dem Zyklus Froyen-lider in ihrem ersten eigenen Gedichtband Kheshvndike nekht [Cheschwan-Nächte]:

tsu dem vel ikh kumen,

ver s'hot der ershter mir mayn froyen freyd gebrakht

un zogn: man,

kh'hob nokh eynem mayn shtiln blik fartroyt

un in a nakht lem im mayn kop geleygt,

ersht hob ikh mayn tsar,

vi binen ongestokhene arum mayn harts gebrakht

3 Vgl. zu den Kiew-Pogromen von 1919 etwa Encyclopedia Judaica (1972:994) und TCHERIKOWER (1965).

$4 \quad$ Kadya Molodowsky (1920): Dorsht und Shtot. In Eygns 2:73-77. 
Die poetischen Passagen der Dichterin Kadya Molodowsky

un hob keyn honik nit oyf lindern mayn vund.

un s'vet der man mir nemen farn tsop,

vel ikh aniderbrekhn zikh oyf beyde fis

un blaybn oyfn shvel vi di farshteynerung fun sdom,

ikh vel di hent aroyfhoybn tsum kop,

vi s'flegt mayn mame ton baym bentshn likht,

nor s'veln mayne finger shteyn vi tsen getseylte zind. ${ }^{5}$

(MoLODOWSKY (1927:12)

Die Situation sozialer und kultureller Umwälzungen, auch im System der Geschlechterverhältnisse, lässt allerdings nicht nur den Gedanken von Autonomie als Ausdruck weiblichen sexuellen Begehrens ihre Froyen-lider durchdringen. Die Gedichte verarbeiten auch das Verlangen nach vielfältigeren Wirkungsmöglichkeiten von Frauen innerhalb gesellschaftlicher und ökonomischer Handlungsräume. In Molodowskys Gedicht-Zyklus Freydke (1935) wird das Bild einer jungen Frau in Warschau, die ihren Lebensunterhalt verdient, indem sie für einen Hungerlohn Eier verkauft, zum Projektionskörper potentieller und unterdrückter Möglichkeiten:

[...]

freydke mayn heldin,

zi volt gekont zayn a gevagter matroz

un firn a shif,

un zayn balebos,

aza hilkhike shtim hot zi.

zi volt gekont haltn in hant a lunet

un zukhn in himl

a nayem planet.

5 ,Ich werde zu dem kommen, / Der mir als erster weibliche Lust bereitet hat / Und sagen: Mann, / Ich habe noch einem anderen meinen stillen Blick anvertraut / Und eines Nachts meinen Kopf neben ihn gelegt, / Nun habe ich meinen Kummer / Wie aufgestachelte Bienen um mein Herz / Und keinen Honig, um meinen Schmerz zu lindern. / Und wenn der Mann mich am Zopf packt, / Werde ich auf die Knie fallen / Und auf der Schwelle verharren wie die Versteinerung von Sodom, / Ich werde meine Hände zu meinem Kopf erheben, / Wie meine Mutter beim Segnen des Lichts, / Allein meine Finger werden stehen, wie zehn abgezählte Sünden. ' - Die Übersetzungen aus dem Jiddischen stammen von der Verfasserin. Dank gilt Ewa Geller für ihre Hilfe bei der Übersetzung ausgewählter Passagen. 
Christina Pareigis

azelkhe likhtike oygn hot zi.

$[\ldots]^{6}$

(MOLODOWSKY 1935:5)

Ihre eigenen politischen und sozialen Ambitionen realisierte Molodowsky neben dem Schreiben als Lehrerin u. a. an einer Grundschule der ,Tsisho ‘?

Die Vermutung liegt nahe, dass ihre Entscheidung für die Emigration 1935 in ihren politischen Aktivitäten gründete. ${ }^{8}$ Fest steht, dass die beiläufig ausgesprochene Einladung zu einem USA-Besuch durch den Leiter des New

,Freydke meine Heldin, / Sie hätte ein mutiger Matrose sein können, / Und ein Schiff steuern, / Und Kapitän sein, / Solch eine starke Stimme hat sie. / Sie hätte ein Teleskop in der Hand halten können, / Und den Himmel / Nach einem neuen Planeten absuchen. / Solch leuchtende Augen hat sie.

7 Der ,Allgemeine jüdische Arbeiterbund (Bund) in Polen und der linke Flügel der ,Poale Zion“ (Jüdische Sozialistische Arbeiterpartei) unterhielten ein Netz säkularer jiddischer Schulen, genannt „Tsentrale yidishe shulorganizatsie“ (Tsisho), das sich bald ganz unter dem Einfluss des ,Bund ' und seiner Ideologie befand. Zur Entwicklung der ,Tsisho“ vgl. PickHAN (2001:236-248).

8 Vgl. Hellerstein (1995:57, Anm. 63): „The reasons for and circumstances of Molodowsky's departure from Poland are ambiguous. According to her autobiography, in Svive 36 (April 1972):60-64, Molodowsky decided rather casually to leave Poland for the United States, where her two sisters and father had settled, by accepting a 1935 invitation from the Sholem Aleichem Folk Institute as a visiting writer. That this ,visit' enforced a three-year separation from her husband renders the casualness of this retrospective account suspect. The gaps in Molodowsky's narration of her departure from her husband and brother, the focus upon marginal details, and the oddly cheery tone all suggest an intentional obfuscation of the actual reasons for her emigration. My sense of this ambiguity was confirmed in a conversation on March 19, 1993, with Anna Gonshor [...] whose thesis-in-progress, ,Kadya Molodowsky: The Warsaw Years', reveals that Molodowsky was very likely motivated to leave Poland because of conflicts in her political activities on the Jewish Left." Zu möglichen Konflikten, die in Molodowskys politischen Aktivitäten gründen, vgl. auch HELLERSTEIN (1995:56, Anm. 48): „Nakhman Mayzl writes in his memoirs that the censor forced Molodowsky to change ,Nakht" (,Night'), a poem in Dzshike Street, about a nighttime police raid so that it referred to the troubles of the Jews in Nazi Berlin, rather than in Warsaw. Molodowsky added 'Berlin, April 1933' to the bottom of the page. Nakhman Mayzl, Geven amol a lebn: dos yidishe kultur-lebn in poyln tsvishn beyde velt-milkomes (Once There Was a Life: Jewish Cultural Life in Poland between the World Wars). (Buenos Aires: Tsentral farband fun poylishe yidn in Argentine, 1951), 291-98. Thanks to Dovid Fishman for this reference." 
Yorker Farlag Matones (Verlag des, Sholem Aleichem Folk Institute") in einer Zeit erfolgte, als Molodowsky sich in einer depressiven Grundstimmung befand, hervorgerufen durch das extreme soziale Elend, mit dem sie tagtäglich während ihrer Arbeit konfrontiert wurde. In ihren viel später entstandenen biographischen Aufzeichnungen kommentiert sie ihre Entscheidung zu emigrieren wie folgt: „I had wandered considerably throughout various cities in Russia and Poland, and to take a stroll to America seemed to me like a hop, skip, and a jump.“ (Zit. nach HELLERSTEIN 1995:37)

Ihr poetisches Werk reflektiert die spezifische Erfahrung der Emigrantin auf vielgestaltige Weise. Einwanderungswilligen aus den osteuropäischen Ländern, allen voran den jüdischen, wurde die Flucht vor sozialer Verelendung und Verfolgung von Seiten der US-amerikanischen Regierung immer schwerer gemacht. Als in den 1920er Jahren zum ersten Mal in der Geschichte der Vereinigten Staaten Einwanderungsbeschränkungen gegen ,Unerwünschte zum (Quoten-)Gesetz erhoben wurden, das die nationale Herkunft zum Kriterium machte, fand dies beim Kongress eine so nachhaltige Zustimmung, dass es auch dann nicht gelockert wurde, als Nachrichten von antisemitischen Gesetzgebungen und Pogromen die deutliche Verschlechterung der Situation der Juden besonders in Deutschland, aber auch in den osteuropäischen Ländern signalisierten. ${ }^{9}$

Wer in die Staaten auswandern wollte, brauchte ein ,Affidavit', die Bürgschaftserklärung einer bereits dort ansässigen Bezugsperson, die positive Auskünfte über intellektuelle und physische Leistungsfähigkeit, moralische Integrität und einschlägige Gründe für eine Einbürgerung, wie die akute Bedrohung im Heimatland, enthalten musste und damit die Funktion eines Einreisevisums hatte.

Im Gedicht Bamit zikh liber khaver, far mir vegn a vize [Bemühen Sie sich, lieber Freund, für mich um ein Visum] aus dem Band In land fun mayn gebeyn. lider [Im Land meiner Gebeine. Gedichte, 1937] wird die zermürbende Wartesaalsituation der Emigranten gezeichnet. Hinter sich die gekappten Wurzeln einer ganzen Erinnerungslandschaft der eigenen Herkunft und Tradition und im Angesicht die Erwartung der Reise in eine unbekannte ,Neue Welt‘:

$9 \quad \mathrm{Zu}$ den Folgen des Emergency Quota Act von 1921 und vor allem des Immigration Act von 1924 vgl. z. B. HERTZBERG (1992:203-222), NGAI (2004) und ZoLBERG (2006). 
es loyft eybik di nakht, di sobvey un mayn harts vundik tsugedrikt tsum kishn.

$[\ldots]$ morgn inderfri, kh'vel vider ondreyen dem zeyger un shraybn briv azelkhe un azetike a shteyger: „ikh shrayb aykh, liber khaver, verter dize, bamit zikh liber dikhter, far mir vegn a vize“. un s'vet a poet a gram ton di velt flink un farsheyt.

- ,,ver vet dort entfern oyf a briv fun epes a natsionaler minderheyt, mir vos hobn shoyn borkheshem..." lakht nisht, mayne vent. ikh bin demoralizirt.

[...] morgn inderfri, $\mathrm{kh}$ 'vel vider ondreyen dem zeyger, azelkhe hastike un bitere teg.

yogt, mayne ferd, ayer farblondzshetn, vaytn geyeg. mayn mantl iz greyt oyfn shtul, mayn groyer shalik oykh.

un mayn ofene gringe valize -

„,bamit zikh liber khaver, far mir vegn a vize...““10

(MolOdOWSKY 1937:42f.)

Die anfangs vielleicht noch empfundene Euphorie bei Bewilligung der ersehnten Papiere wurde schnell unterlaufen von der Ahnung, dass das Versprechen einer offenen und pluralistischen Schmelztiegelgesellschaft nur um den Preis der Aufgabe der mitgebrachten Eigenarten und Traditionen sowie der Anpassung an die herrschende Ideologie des ,success“ einzulösen sei. So verweisen in Molodowskys Gedicht mit der zweideutigen Überschrift „Ar-

10 ,Es läuft endlos die Nacht, die Subway und mein Herz / wund, gedrückt ins Kissen / [...] / Morgen früh werde ich wieder die Uhr aufziehen / Und Briefe dieser Art schreiben: / „Ich schreibe Ihnen, lieber Freund, diese Worte, / Bemühen Sie sich, lieber Dichter, für mich um ein Visum.“ / [...] Und ein Dichter wird geschickt und schamlos einen Reim an die Welt schreiben / „Wer wird denn einen Brief beantworten / [geschrieben im Namen] von irgendeiner nationalen Minderheit, / Wir, die wir gottseidank schon..." / Lacht nicht, meine Wände. / Ich bin demoralisiert. / [...] / Morgen früh werde ich wieder die Uhr aufziehen, / Was für hastige und bittere Tage. / Jagt, meine Pferde, eure verirrte, weite Jagd. / Mein Mantel liegt auf dem Stuhl bereit, / Mein grauer Schal auch. / Und mein offener, leichter Koffer - / „Bemühen Sie sich, lieber Freund, für mich um ein Visum...““ 
opgekumen“" (,Heruntergekommen') inmitten der farbenprächtigen heiteren Schilderung der Ankunft in New York synästhetische Brechungen auf eine Spur von Angst und Gewalt:

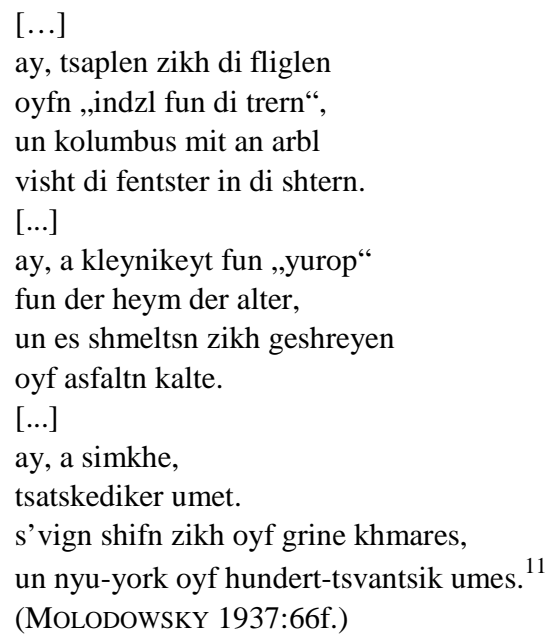

Bedingungen einer historischen Wirklichkeit konstituieren im Gedicht das gewalttätig anmutende Bild der personifizierten Stadt New York, die sich auf der zur amorphen Schmelztiegelmasse und damit zur undifferenzierten Einheit toter Dinglichkeit verwandelten Nationen-Zahl „wiegt“. Die Umkehrung der Konnotationen , menschlich ` - dinglich ' führt in eine nach Gesetzen der Abhängigkeiten organisierte Welt, in der der einzelne Mensch auf Kosten seiner ausgeprägten nationalen Kulturformen im Begriff ,hundertzwanzig Nationen" aufgeht und in der er der Macht der Gegenständlichkeit buchstäblich unterworfen ist. Sie führt in eine Welt, deren Zugang das schmerzkonnotierte Symbol Ellis Island, „Insel der Tränen“, ist. Die SchmelztiegelTheorie implizierte den ständigen Wandel der amerikanischen Gesellschaft, allerdings in Gang gesetzt durch die allmähliche Einebnung kultureller und sprachlicher Differenzen: Ein Amerikaner zu sein, bedeutete die Identi-

11 ,Ei, es zucken die Flügel / Auf der „Insel der Tränen“, / Und Kolumbus putzt mit dem Ärmel / Die Fenster in den Sternen. / [...] / Ei, eine Kleinigkeit aus „Europe“ / Aus der alten Heimat, / Und es zerschmelzen Schreie / Auf dem kalten Asphalt. / [...] Ei, eine Freude / lustige Traurigkeit / Schiffe wiegen sich auf grünen Wellen, / Und New York auf hundertzwanzig Nationen. 
fikation mit der amerikanischen Gesellschaft und dem , weißen angelsächsischen Protestanten' als deren Repräsentanten.

Die Mehrzahl der jungen jüdischen Intellektuellen empfand Amerika nicht als Schmelztiegel, nicht zuletzt, weil ihnen die Gesellschaft die Verschmelzung verweigerte, aber auch, weil viele ahnten, dass im Falle einer ,Verschmelzung ' der auf Herkunft und Kontinuität gründende Teil ihrer Zugehörigkeit verlorenginge. Unter den osteuropäisch-jüdischen Schriftstellern war die Zerrissenheit zwischen der Suche nach einem Ort innerhalb der amerikanischen Kultur im Bewusstsein der Marginalität und der Abwehr der drohenden kulturellen Nivellierung besonders ausgeprägt. So war neben dem Bemühen um Akkulturierung eine Antwort auf ihre Frage nach einem Platz in dieser Gesellschaft die bewusste Entfremdung durch die Kultivierung ihrer Marginalität und der Aufbruch (aus) der traditionellen Überlieferung.

Die Sozialerfahrungen zwischen den Welten ließen die Schriftsteller der 1930er Jahre sich mehr und mehr aus den Schatten der ,Poets of Yiddishkayt ${ }^{\star}$ wie Scholem Aleichem lösen, die sich um die Jahrhundertwende einer traditionellen Poesie gewidmet hatten, angefüllt mit Elementen aus der jiddischen Folklore und ,authentischen ' Portraits jüdischen Lebens in Osteuropa. Während ihre Geschichten meist dann abbrechen, wenn ihre Protagonisten den Mikrokosmos ,Schtetl 'verlassen, führen die Fluchtbewegungen in den Werken der jüngeren Dichtergeneration oftmals aus dem traditionellen Judentum und der Tradierungsbewegung selbst heraus. ${ }^{12}$

12 Die Entwicklung der Schriftsteller, die am Jiddischen festhielten, sich aber von den früheren Schriftstellerautoritäten emanzipieren wollten, lässt sich an den Programmen der literarischen Avantgarde ablesen. Vor allem in der Zeit zwischen den Weltkriegen folgte eine Schule der nächsten: Es begann mit ,Di yunge', einer Bewegung, die gleichzeitig in Russland, Polen und den USA entstand. Die Texte, die aus ihr hervorgingen, verschmolzen u. a. Elemente der Romantik, des deutschen Expressionismus und des russischen Symbolismus. Propagiert wurde der Ausdruck von Subjektivismus in einer Dichtung, die zugleich stark von Gesellschaftskritik geprägt war. - Die Mitglieder der in den 1920er Jahren in den USA gegründeten Gruppe „In zikh“ plädierten in ihrem Manifest zur introspektivistischen Lyrik für freies Versmaß, gleichberechtigten Ausdruck von Gefühl und Rationalem sowie Experimentierformen, die den ,natürlichen Rhythmus des gesprochenen Jiddisch reflektieren sollten. Ziel der ,Champions of Yidishkayt" war es, die jiddische Lyrik in der modernen Literaturlandschaft zu etablieren. Vgl. AARON (1990:1-19) und zum Selbstverständnis zeitgenössischer jiddischer Schriftsteller in den USA auch FINKELSTEIN (2001:35-54, insbesondere 36-39). 
Als Molodowsky 1935 Ellis Island in Richtung New Yorker Ufer verließ, steuerte sie zugleich der Ankunft inmitten dieses Spannungsfeldes von Auslöschung, Bewahrung und Erneuerung entgegen. Ihre Texte akzentuieren ab jetzt verstärkt die Sprache als Trägerin der Tradition und als Schöpferin von Wirklichkeit, als Vermittlerin von Welten und als Stifterin von Identität wie Differenzen, solange es Sprachen in der noch existierenden Vielfalt gibt. Die Anzeichen eines drohenden Verlustes des Jiddischen durch die amerikanischen gesellschaftlichen Verhältnisse, in die all jenen der Zutritt verweigert wird, die die Sprache der Herrschenden nicht beherrschen, konstituieren ab jetzt ein Zentralmotiv in Molodowskys Dichtung. Den Prozess eines mit seiner Entwurzelung sich vollziehenden Verlustes sprachlichen Gedächtnisses artikuliert das Gedicht Oysyes [Buchstaben]:

in bronks, in bruklin un nyu york-siti,

hobn mayne shvesterkinder kromen.

zibn shvesterkinder mit zibn kromen, vi gebotn.

bizneslayt mit lange tsetlen fun bankrotn.

un mayn familye-nomen hot a kuk geton oyf mir fun zeyere shildn

mit a blik - a fremdn un a vildn.

es hot der flamediker „mem“ (fun moyshe un fun marks)

gehipet oyf a grinem fisl.

der ,alef“ hot blank gevunkn tsu der gas.

der „lamed“ hot oysgeshlayft zikh vi a tlie.

un s'hot der ,,alef-beys“" geshrien in ayzernem gebrozg fun shtot:

- bankrot, bankrot un nokhamol bankrot.

nor untern shild zaynen der feter mikhl un di mume sore

breyt gevorn, keyn ayen hore.

$\mathrm{zi}$ - a bloe zaydene fas,

un er - a groye, shtolene sprunzshine.

un di kinder:dzshuli, bitris, meks un karoline,

hobn shtolts getrogn oyfn pleytse oysyes vi shtern,

tsu velkhn kortn klub un boy-skaut zey gehern. ${ }^{13}$

(MOLODOWSKY 1937:75)

13 ,In der Bronx, in Brooklyn und in New York City, / Haben meine Cousins Läden. / Sieben Cousins mit sieben Läden, wie Gebote. / Businessleute mit langen Bankrott-Listen / Und mein Familienname sah auf mich herab von ihren Schildern / Mit einem fremden und wilden Blick. / Der flammende „Mem“ (von Mose und Marx) / Sprang auf einem grünen Fuß. / Der „Alef“ winkte strahlend zur Straße herab. / Der „Lamed“ schlang sich zu einem Knoten wie ein Henkersseil. / Und der „Alef-Beys“ schrie in das eiserne Gebraus der Stadt: / - Bankrott, Bankrott und noch einmal Bankrott. / Aber unter dem Schild sind der Onkel 


\section{Christina Pareigis}

Ausgehebelt aus ihrer kollektiven Geographie, beginnen die Buchstaben des „Familiennamens“ „Molodowsky“, dem Zugehörigkeitszeichen im gemeinschaftlichen Kontinuum der Generationen, einen „fremden“ und „wilden“ Tanz. In den Kontext westlicher Industrienation gestellt, bekommen sie eine individualisierte Identität verliehen. In einer Art Vulgär-Gematrie mischt sich die kollektive Erinnerung in Form von mit den jiddischen Anfangsbuchstaben assoziierten religiösen und säkularen Zeichen (,Moses“ und „Marx“) mit den sprachlichen Zeichen einer an merkantilen Zwecken orientierten Kultur, deren Grellheit sich bis zur dreifachen Bankrotterklärung steigert.

Buchstaben wie Menschen werden beim Verlassen ihrer Geographie zu Subjekten einer Geschichte in dem Maß, wie sie von den kapitalistischen Macht-Dispositiven erfasst werden, die eine selbst verantwortete Biographie, individuelle Autonomie, wirtschaftliche Produktivität etc. abfragen. Die Signale der Zugehörigkeit zu den Institutionen, die den Prozess jener Erfassung stützen, trägt die zweite Generation bereits in Form von amerikanischen Buchstaben am Körper: „Auf dem Rücken wie Sterne““. ${ }^{14}$ Das Fortereignen der kollektiven osteuropäisch-jüdischen Tradition wird in amerikanische Geschichte und Individualität umgeschrieben. An die Stelle einer gemeinschaftlichen wie erinnerungsstrategischen Überlieferungsbewegung, wie sie die jiddische Sprache verkörpert, treten die Register einer repräsentativen Identität, die in der Existenz als „blaues, seidenes Fass“ und als „graue, stählerne Sprungfeder“ endet.

Sprache hat ein mindestens doppeltes Gesicht, solange Differenz Diskriminierung bedeutet und Sprache als Mittel der oft auch sozialen Unterdrückung

Michl und die Tante Sore / in die Breite gegangen, gut bekommen soll's ihr. / Sie - ein blaues seidenes Fass, / Und er - eine graue, stählerne Sprungfeder. / Und die Kinder: Julie, Beatrix, Max und Carolyn, / Trugen stolz auf dem Rücken Buchstaben wie Sterne, / Für die Karten-Clubs und Pfadfinder-Gruppen, denen sie angehören.

14 Vgl. hierzu auch Hellerstein (1995:78): Hellerstein verweist auf die ambivalente Funktion des Verhältnisses von jiddischen und englischen Buchstaben in Molodowskys Gedicht: „Disembodied letters of the English alphabet [...] indicate the degree to which they [the immigrants' American-born children - Ch.P.] have successfully assimilated in American culture. Thus, while the Yiddish letters spelling out a fragment of the family name point toward the immigrant generation's failure in America, the English letters on the sleeves of children with American names seem to point in the opposite direction. But, in fact, the letters spell nothing. Unanchored signs in the poem, floating free of language, they point beyond exile." 
dient oder den Unterdrückten Barriere bleibt - eine Erkenntnis, die Molodowsky immer wieder in ihren Texten thematisiert. Englisch ist das Entréebillett in die Bereiche der Arbeit und der sozialen Beziehungen Nordamerikas; es zu erlangen, bedeutet die Aufgabe der mitgebrachten Tradition, verkörpert im alltäglichen Gebrauch der jiddischen Sprache.

MoLODOWSKYs Prosastück Fun lublin biz nyu york. togbukh fun Rivke Zilberg [Von Lublin bis New York. Das Tagebuch der Rivke Zilberg, 1942], das Tagebuch eines jungen Mädchens, das zur Zeit der Okkupation Lublins durch die deutschen Nazis nach New York emigriert, reflektiert immer wieder die Beziehungen zwischen Sprache und Macht und wie sich Macht durch Sprache gefügige Untergebene schafft. Als Rivke bei der Ankunft ihren Verwandten vorgestellt wird, offenbart sich bei jenen der bereits vollzogene Sprachenwechsel, zunächst in der Begrüßung durch die Cousine: „zi hot tsu mir haynt aroysgeredt a por verter. ,vi filstu?` un ,du bist mayn kozin`, mer ken zi nit redn keyn yidish, zogt zi.“ (MOLODOWSKY 1942:5) ${ }^{15}$ Von ihrer Umwelt gedrängt, sich doch einen ,dzshob` zu verschaffen und damit den Grundstein für jene individualisierte, vom Band der Familie unabhängige amerikanische Biographie zu legen, stellt sich das Bewusstsein über den Zusammenhang von Sprache und merkantilem Zweck ein:

[...] tsen doler a vokh makht zi derfun nor men muz kenen english. az eyne, an opreyterke git a ruf: „kam over hir“ (kum aher), muz men vizn vos dos meynt, un az a tsveyte git a ruf „teik it evei“ (nem es avek!), muz men vizn vos zi meynt. un az an opreyterke iz ,noyrvez“ (nervez) un tseshrayt zikh: ,,am sik end tayerd of de dost!“ (ikh bin toyt mid fun dem shtoyb) muz men oykh farshteyn vos dos meynt. (MOLODOWSKY 1942:43f.)

Der Text deckt verborgene Wechselwirkungen zwischen Sprache und Macht und nur sprachlich zu vermittelnder Ideologie auf. Die erlernten englischen Phrasen quittieren die Zustände in der Welt der Fabrikarbeiter und beschränken die ökonomischen Tatsachen auf das, was sie eben nicht nur sind, wie es der Jargon kalkulierter Knappheit vormachen will.

15 ,Sie hat heute ein paar Worte mit mir gesprochen. „Wie geht es Dir?“ und „Du bist meine Cousine“, mehr Jiddisch kann sie nicht, sagt sie."

16 ,[...] zehn Dollar die Woche verdient sie, man muss nur Englisch können. Wenn eine Aufseherin ruft: „Come over here“ (Komm her), muss man wissen, was das heißt, und wenn eine zweite ruft: „Take it away“ (Nimm es weg!), muss man wissen, was sie meint. Und wenn eine Aufseherin „nervous“ (nervös) ist und schreit: „I'm sick and tired of the dust!“ (Ich bin todmüde vom Staub), muss man auch verstehen, was das heißt. ' 
Entwurzelt und ausgegliedert sucht Rivke nach dem, was den Kontakt zum anderen ermöglicht: Es ist die Sprache. Doch hier ist es die Sprache der angepassten Verwandten, die diesen die Überlegenheit sichert. Rivke und ihre Geschichte werden in der Rede ihrer Umgebung nicht einbezogen. Dennoch kommen im diffusen Verstehen der über ihren Kopf hinweg geführten Gespräche Spuren von Verdrängtem an die Oberfläche und erzeugen in der belanglos scheinenden Debatte um ,success-stories ' (wer ist der größte ,Star Amerikas?) unterschwellige Spannungen, aggressiv aufgeladen vom Konkurrenzhabitus der Einzelnen:

vos zey hobn alts geredt hob ikh kimat gornit farshtanen. ikh hob bloyz gezen, az ale zaynen royt, ale zaynen beyz, ale brenen vi fayer. mir hot zikh gedakht, az zey krign zikh gor vegn andere zakhn, vos lign zey tifer in hartsn, vi edi kentor un glen miler. ver veys? un efsher ligt zey take glen miler azoy tif in der neshome? vos ken men visn, az zey redn english? (MOLODOwSKY 1942:50) ${ }^{17}$

In der Gestalt der Emigrantin Rivke wird die Erfahrung artikuliert, Bürgerin zweier Sprachwelten zu sein, ohne je wieder die soziale Einbindung in eine der beiden Welten zu erreichen. Vielleicht auch deshalb nicht, weil die sprachliche Tradition mitsamt ihres Gedächtnisses sich doch nicht so leicht auf das Rudiment einer gekappten Herkunft reduzieren lässt, wie die Mächtigen der Arbeitswelt und die assimilierten Verwandten es erwarten. Während die ,londrinitshke“ (,Wäscherin'), bei der Rivke schließlich einen Job findet, tagsüber ausschließlich Englisch und in sprachverkürztem Anweisungsjargon spricht, beginnt sie in einer Nacht der Wunder auf Jiddisch von ihrer russischen Herkunft, von ihrer akademischen Ausbildung in Warschau und ihrer Liebe zu Leo Tolstoi zu erzählen. Während bei Tag das jiddische Gedächtnis schweigt, bringt die Nacht es zum Sprechen, wenn nämlich die lediglich zeitweilig verdrängte Dynamik des gesprochenen Jiddisch jener (Sprach-)Welt Widerstand leistet, die sie am liebsten zum Verstummen bringen würde.

Auch in Molodowskys späteren Schriften finden sich Anzeichen plakativer Kritik des amerikanischen Materialismus, moralischer Korruption und der Assimilation, aber auch der Kommerzialisierung des Literaturbetriebs, der sich bloß in seiner wirtschaftlichen Effizienz durch Literaturproduktion in

17 ,Über was sie alles geredet haben, habe ich fast gar nicht verstanden. Ich habe bloß gesehen, dass sie alle rot und böse sind und wie Feuer brennen. Ich dachte, dass sie sich um andere Dinge streiten, die ihnen tiefer am Herzen liegen, als Eddie Cantor und Glenn Miller. Wer weiß? Und vielleicht liegt ihnen Glenn Miller wirklich so tief in der Seele? Wie kann man das schon wissen, solange sie Englisch reden?' 
englischer Sprache legitimiert sieht. ${ }^{18}$ Jiddisch als Medium jüdischen Bewusstseins, Ethik und Identiät proklamiert Molodowsky als Gegenmittel gegen die kritisierten Erscheinungen. In ihrem viel später entstandenen Essay Opgeshlagn fun beyde bregn. vegn yidn vos shraybn oyf fremde shprakhn [Von beiden Ufern weggetrieben. Über Juden, die in fremden Sprachen schreiben, 1963] schreibt sie ihre Sprachkritik fort. Darin vergleicht sie jüdische Schriftsteller, die sich vom Jiddischen lossagen, mit ,vaser blumen on vortslen vos vern mitgeshlept mitm shtroml“ (,Wasser-Blumen ohne Wurzeln, die vom Strom mitgetrieben werden'). Jiddisch ist das Gedächtnis der Generationen: „der shrayber dertseylt nit bloyz vos er filt oder trakht, oder zet, nor ale khaloymes fun tate-mame, zeyde-bobe un oves-avoyseynu iz er poyser-kholem. er iz der treger fun a sakh gilgulim." (MOLODOWSKY 1963, zit. nach KLEPFISZ 1994:34f.) ${ }^{19}$ Jene tradierte Fähigkeit, die Träume der vorangegangenen Generationen zu lesen, geht mit dem Verlust des in Sprache lebendigen Gedächtnisses verloren: „,ven men shraybt oyf a fremder shprakh falt aroys der shprakh-zikorn un afile ven men nemt a idishe motiv kumt er aroys barves un naket un on a neshome." (MOLODOWSKY 1963, zit. nach KLEPFISZ 1994:34) ${ }^{20}$

Der Text in Jiddisch vermittelt nicht einfach die Bedeutung, die sich in einem linguistischen Körper wie eine flache Struktur aus Signifikanten, Grammatik und Syntax präsentiert. Er trägt das „,shprakh-zikorn“ (,Sprach-Gedächtnis“) mit sich, die destillierte Essenz jüdischen Lebens und dessen kollektive Seele: Sprache ist alles. Nicht, was geschrieben wird, ist ausschlaggebend, sondern dass in Jiddisch geschrieben wird. Der jiddische Text ist ein jüdischer per se, dagegen ist ein nichtjiddischer Text mit jüdischen Themen etwas „Seelenloses“, so lautet die radikale Gleichung Molodowskys.

Die Sprach- und Sozialkritik in Molodowskys essayistischen und poetischen Texten verweist auf ein grundlegendes strukturierendes Element ihrer Poetik: den drohenden Verlust von Sprachfähigkeit, repräsentiert im Jiddischen als der Dichterin individuelles sprachliches und gleichzeitig im Kollektiv ihrer

18 Zur Publikationslage der jiddischen Schriftsteller vgl. Howe (1976:440-445).

19 ,Der Autor erzählt nicht allein von dem, was er fühlt, denkt oder sieht, sondern er deutet auch alle Träume der Eltern, Großeltern und Vorfahren. Er ist Träger vieler Seelenwanderungen. ${ }^{*}$

20 ,Schreibt man in einer fremden Sprache, verschwindet daraus das SprachGedächtnis, und selbst wenn man in ihr ein jüdisches Motiv verarbeitet, erscheint es barfuß, nackt und seelenlos. 
Herkunft verankertes Gedächtnis. Verschärft wird das Bewusstsein jenes Verlustes angesichts der Lage der Juden in Europa. Die ersten konkreten Nachrichten über den systematischen Mord erzeugten in Molodowsky das panische Bewusstsein einer doppelten totalen Vernichtung, nämlich der Menschen mitsamt ihren sprachlichen Zeugnissen, und zugleich die Verzweiflung über die weitgehende Ignoranz gegenüber jenem Wissen in der amerikanischen Bevölkerung. Unter den Juden Amerikas trafen diese Nachrichten auf eine gedrückte Atmosphäre, gekennzeichnet von einem Antisemitismus, bedrohlicher als jemals zuvor in der amerikanischen Geschichte, und den noch immer spürbaren Folgen der Wirtschaftskrise. Zudem begann die jiddische Einwandererkultur langsam zu verschwinden. Nur amerikanisches Englisch, nicht aber Jiddisch drückte amerikanische Kultur gültig aus.

In Molodowskys Wahrnehmung wurde die Bedrohlichkeit der Informationen aus der Alten Welt von der amerikanischen Gesellschaft in dem Maß ignoriert und verlor sich im Tiegel der Verschmelzung, wie sie von ihren jüdischen Mitgliedern verlangte, in der Gleichheit massenhaft gleicher Unverbindlichkeit aufzugehen, wenn sie nicht ausgegrenzt werden wollten. Eine Situation, die in ein ,miting“ der Lubliner „landsmanshaft“ im Togbukh führt: Als Rivke glaubt, in den von der Vorsitzenden auf Jiddisch geäußerten, mit englischen Anweisungen kommentierten Sprichwortsentenzen gehe es um einen Kuchenbackwettbewerb als Vorbereitung auf das lustige Purimfest, wird sie eines Besseren belehrt:

nor men hot mir derklert, az dos darf zayn a zamlung far di milkhome korbones. es iz geven gants freylekh. men hot zikh gekhokhmet un gegesn keik (tort). in amerike est men zeyer a sakh „keik“. abi vos iz „keik“. a kart padhi - iz keik, a geburtstog - iz „keik“, a zamlung far di milkhome gelitene - iz „keik“. (MoLODOWSKY $1942: 21)^{21}$

Die Bindekraft ganzer Traditionen verdünnt sich zum ,small talk', der sich den ethischen Diskurs mit einem Stück Käsetorte einverleibt und schließlich zum Verstummen bringt. Zwischen Kultur und Esskultur bestehen mehr als nur äußere Beziehungen. Von deren Bedeutung bleibt dort wenig, wo die Freiheit zu maßlosem Verzehr auch dann fast ausschließlich zählt, wenn die

21 ,Schließlich hat man mir erklärt, dass es sich um eine Sammlung für Kriegsopfer handelt. Es ging sehr fröhlich zu. Man hat Witze gerissen und cake (Torte) gegessen. In Amerika isst man sehr viel „cake“. Was ist nun „cake“. Eine Karten-Runde - ist cake, beim Geburtstag - gibt es „cake“, bei einer Sammlung für die Kriegbeschädigten - wird auch ,cake“ serviert. 
Ungeheuerlichkeit der Nachrichten aus ,der alter heym“ über das Fortschreiten der Vernichtung selbst ins Maßlose steigt.

Dem inneren und äußeren Leben der jüdischen Bevölkerung Amerikas wohnte in jenen Jahren eine Schizo-Wirklichkeit inne. Zu Hause in Amerika: allmähliches Zurruhekommen in Zufluchtssicherheit, im Gefühl relativer ökonomischer und sozialer Stabilität, trotz Antisemitismus und nur langsam sprießender Wirtschaft; zu Hause in Europa: die Judenverfolgung, die Todeslager.

Die radikale Unbedingtheit, mit der Molodowsky Jiddisch als Lebensvoraussetzung für die osteuropäische jüdische Kultur proklamierte, wurde zerbrechlich durch die Erkenntnis, dass das sprachliche Gedächtnis keinen Haltepunkt mehr findet, dass Jiddisch sich als versteinerte Erinnerung in der kollektiven Gedächtnisdeponie ablagert, weil seine Sprecher sich entweder der Sprache ihres neuen geographischen Kontextes zuwenden oder unwiederbringlich aus ihrer ursprünglichen Geographie ausgelöscht werden. Ihre poetischen Passagen akzentuieren die Schwellen-Situation der Einwanderer, den Druck der Schmelztiegelgesellschaft und vor allem immer wieder Konstellationen von Sprache, Zugehörigkeit und Macht sowie Jiddisch als kollektives Sprachgedächtnis. Die Anzeichen des Verlustes jenes Gedächtnisses durch die Differenzen absorbierende amerikanische Gesellschaftssituation konstituieren ein Zentralmotiv ihrer Dichtung seit den 1930er Jahren. Sie gibt poetisches Zeugnis von Differenzen zwischen Topographien und Zeiten, zwischen Sprachen und Traditionen und einem von seiner geographischen Herkunft abgeschnittenen kulturellen und sprachlichen Gedächtnis - und das, noch bevor ein Bewusstsein der drohenden Vernichtung am Ort jener Herkunft das Gedenken in ihrer Poesie formen konnte.

\section{Literatur}

Aaron, Frieda W. (1990): Bearing the Unbearable. Yiddish and Polish Poetry of the Ghettos and Concentration Camps. Albany/New York.

Encyclopedia Judaica (1972). Bd. 10. Jerusalem.

Finkelstein, NoRman (2001): Jewish American Modernism and the Problem of Identity. In: FinKelstein, NoRman: Not One of Them in Place. Modern Poetry and Jewish American Identity. Albany/New York. 
FISHMAN GONSHOR, ANNA (1997): Kadye Molodowsky. In: Literarishe bleter, 19251935. [Kadya Molodowsky in Literarische Blätter]. Annotated Bibliography (M.A. thesis, McGill University, Montreal, Internetveröffentlichung: http://digitool.library. mcgill.ca/R/?func=dbin-jump-full\&object_id=28054\&local_base=GEN01-MCG02, 1997).

Harshav, Benjamin (1995): Hebräisch. Sprache in Zeiten der Revolution. Aus dem Englischen übersetzt von Christian Wiese. Frankfurt (M).

Hellerstein, KATHRYN (1995): In Exile in the Mother Tongue: Yiddish and the Woman Poet. In: Henderson, MAE (ed.): Borders, Boundaries and Frames. New York/London.

HertzBerg, Arthur (1992): Shalom, Amerika! Die Geschichte der Juden in der Neuen Welt. Aus dem Amerikanischen von Sylke Tempel. Frankfurt (M.)/Wien.

Howe, IRving (1976): World of Our Fathers. New York.

KlepFisz, Irena (1994): Di mames, dos loshn. The Mothers, the Language: Feminism, Yidishkayt, and the Politics of Memory. In: Bridges 1:12-47.

Molodowsky, KadYA (1927): Kheshvndike nekht. [Cheschwan-Nächte]. Vilne [Wilna].

- (1935): Freydke. Varshe [Warschau]. (=Literarishe bleter [Literarische Blätter])

- (1937): In land fun mayn gebeyn. lider. [Im Land meiner Gebeine. Gedichte]. Chicago.

- (1942): Fun lublin biz nyu-york. togbukh fun Rivke Zilberg. [Von Lublin bis New York. Das Tagebuch der Rivke Zilberg]. New York.

- (1963): Opgeshlogn fun beyde bregn. vegn yidn vos shraybn oyf fremde shprakhn. [Von beiden Ufern weggetrieben. Über Juden, die in fremden Sprachen schreiben]. In: Svive. [Umfeld]. Zit. nach KLEPFISZ (1994:34f.).

- (1965-1974): Mayn elterzeydns yerushe. [Das Erbe meines Urgroßvaters]. In: Svive. Zit. nach HELLERSTEIN (1995).

- (1999): Papirene Brik. geklibene lider fun Kadye Molodovski. Paper Bridges. Selected Poems of Kadya Molodowsky. Introduced and translated by Kathryn Hellerstein. Detroit.

NGaI, MAE M. (2004): Impossible Subjects. Illegal Aliens and the Making of Modern America. Princeton.

PickHAN, GERTRUd (2001): „Gegen den Strom“. Der Allgemeine Jüdische Arbeiterbund „Bund“ in Polen 1918-1939. Stuttgart/München u.a. (=Schriften des SimonDubnow-Instituts Leipzig).

Ravitsh, MeleKh (1945): Kadya Molodowsky. In: Mayn leksikon. yidishe dikhter, dertseyler; dramaturgn in poyln tvishn di tsvey groyse velt milkhomes. [Mein Lexikon. Jiddische Dichter, Erzähler, Dramatiker in Polen zwischen den beiden großen Weltkriegen]. Bd. 1. Montreal, 122-124.

Shapiro, Edvard Steven (ed.) (2008): Yiddish in America: Essays on Yiddish Culture in the Golden Land. Scranton/PA.

SOYER, DANIEL (1997): Jewish Immigrant Associations and American Identity in New York, 1880-1939. Cambridge (Mass.)/London. 
Die poetischen Passagen der Dichterin Kadya Molodowsky

TCHERIKOWER, ElIAS (1965): Di ukrainer pogromen in yor 1919. [Die ukrainischen Pogrome im Jahr 1919]. New York.

WEINREICH, URIEL (1990): Modern english-yidish yidish-english verterbukh. [Modernes Englisch-Jiddisch Jiddisch-Englisch Wörterbuch]. New York.

Zolberg, Aristide R. (2006): A Nation by Design: Immigration Policy in the Fashioning of America. Cambridge (Mass.). 